\title{
Shunted-Josephson-junction model. I. The autonomous case
}

Belykh, V. N.; Pedersen, Niels Falsig; Sørensen, O. H.

\section{Published in:}

Physical Review B

Link to article, DOI:

10.1103/PhysRevB.16.4853

Publication date:

1977

\section{Document Version}

Publisher's PDF, also known as Version of record

Link back to DTU Orbit

\section{Citation (APA):}

Belykh, V. N., Pedersen, N. F., \& Sørensen, O. H. (1977). Shunted-Josephson-junction model. I. The autonomous case. Physical Review B, 16(11), 4853-4859. https://doi.org/10.1103/PhysRevB.16.4853

\section{General rights}

Copyright and moral rights for the publications made accessible in the public portal are retained by the authors and/or other copyright owners and it is a condition of accessing publications that users recognise and abide by the legal requirements associated with these rights.

- Users may download and print one copy of any publication from the public portal for the purpose of private study or research.

- You may not further distribute the material or use it for any profit-making activity or commercial gain

- You may freely distribute the URL identifying the publication in the public portal

If you believe that this document breaches copyright please contact us providing details, and we will remove access to the work immediately and investigate your claim 


\title{
Shunted-Josephson-junction model. I. The autonomous case
}

\author{
V. N. Belykh* \\ Electronics Laboratory, The Technical University of Denmark, DK-2800 Lyngby, Denmark
}

\author{
N. F. Pedersen and O. H. Soerensen \\ Physics Laboratory I, The Technical University of Denmark, DK-2800 Lyngby, Denmark
}

(Received 26 October 1976)

\begin{abstract}
The shunted-Josephson-junction model: the parallel combination of a capacitance, a phase-dependent conductance, and an ideal junction element biased by a constant current, is discussed for arbitrary values of the junction parameters. The main objective is to provide a qualitative understanding of the junction behavior in different regions of the parameter space. Approximate formulas are given for the parameter-space decomposition into regions of qualitatively different junction behavior corroborated by the associated-phase plane portraits and also approximate expressions for the corresponding dc currrent-voltage curves are presented. The case with a time-dependent monochromatic bias current is treated in a similar fashion in the companion paper.
\end{abstract}

\section{INTRODUCTION}

Most of the recent theoretical work directed towards applications of the Josephson junctions has been based on the shunted-junction model, with good reason, since this model seems to account very well for a large variety of experimental results. Conceptually this model is the simplest possible consisting of a parallel combination of currentcarrying elements specified as follows: (i) the ideal Josephson element carrying a current $I_{1}$ $=I_{0} \sin \phi$, where $I_{0}$ is the junction critical current, and $\phi$ is the phase difference between the macroscopic wave functions on either side of the junction, (ii) a linear conductance $G$ giving rise to a current $I_{2}=G V$, (iii) possibly a phase-dependent current of the form $I_{3}=\epsilon G \cos \phi V$, and (iv) a displacement current of the form $I_{4}=C d V / d t$. The phase difference is related to the voltage $V$ across the junction by $d \phi / d t=2 \mathrm{eV} / \hbar$, a relation involving only fundamental constants.

In almost every case the junction will appear to be current controlled, which is the origin of most of the problems concerning this device. If an external current source is coupled to the junction this current must equal the sum of currents $I_{1}$ through $I_{4}$, and if the externally applied current contains a dc component $I_{\mathrm{dc}}$ and an ac component at frequency $\omega / 2 \pi$ and amplitude $I_{\mathrm{ac}}$, the system behaves according to the nonlinear second-order differential equation

$$
\begin{aligned}
I_{\mathrm{dc}} & +I_{\mathrm{ac}} \sin \omega t \\
& =(\hbar C / 2 e) \ddot{\phi}+(\hbar G / 2 e)(1+\epsilon \cos \phi) \dot{\phi}+I_{0} \sin \phi,
\end{aligned}
$$

where the voltage has been eliminated using

$$
V=\hbar \dot{\phi} / 2 e \text {. }
$$

Equation (1) has been discussed in numerous publications with and without a time-dependent bias current. In neither case is the equation analytically solvable. Accordingly, a variety of approximate methods have been used in parallel with calculations on analog and digital computers. Assuming a dc bias current $\left(I_{\mathrm{ac}}=0\right)$, the simplest case $C=0, \epsilon=0$ is readily integrated. Stewart ${ }^{1}$ and McCumber ${ }^{2}$ discussed Eq. (1) with $\epsilon=0$ and $C \in(0, \infty)$ as a parameter. They found by numerical calculation that the dc current-voltage relation for the device in a range of bias currents $I_{\mathrm{dc}}<I_{0}$ was double valued, and that the amount of hysteresis increased with increasing $C$. Schlup ${ }^{3}$ generalized their results to include a nonzero $\epsilon \in(-1,+1)$ approaching the problem using perturbation theory combined with numerical calculations.

A different line of approach has been to bring the equation into an analytically solvable form by modifying the individual terms, i.e., either by approximating the Josephson term $I_{0} \sin \phi$ by other periodic functions of $\phi,{ }^{4}$ or by replacing the linear conductance term $G V$ by the nonlinear form $G V^{n}$ where $n=2,,^{5,6}$ or $n \rightarrow \infty .{ }^{6}$ The obtained results have been qualitatively similar independent of how the problem was approached.

To date there has been no rigorous theoretical justification of the shunted-junction model defined by Eqs. (1) and (2). Josephson's original papers,${ }^{7}$ dealing with the thin-film tunnel junction, does have current components of the form stated here, only the coefficients $I_{0}, G$, and $\epsilon$ are dependent on junction voltage. ${ }^{8}$ This dependence may be neglected provided the voltage stays small compared to the energy-gap voltage of the individual superconductors. A more serious limi- 
tation is that the current terms were derived assuming voltage bias of the junction, which contrasts with the current-biased situation most often encountered in practice. Current contributions of the same general form as in Eqs. (1) and (2) have been derived also for the thin-film microbridge ${ }^{9}$; however, also for this case the above mentioned objections are valid. The third commonly used Josephson-junction structure, the point contact, may have partly tunnel junction and partly microbridge character. Nevertheless, the shuntedjunction model gives a good qualitative description of all these different structures. Accepting the model as basically phenomenological, it becomes important to understand the dependence on the model parameters, and from comparison with experiments, to extract their values. Presently, it will be particularly interesting to determine the dependence on $\epsilon$ since the existing theories for tunnel junctions ${ }^{8}$ and microbridge ${ }^{9}$ disagree with respect to the $\operatorname{sign}(\epsilon>0$ and $\epsilon<0$, respectively) and all experiments so far seem to favor a definite sign (minus) ${ }^{10}$ independent of on which device the experiment is made.

In this paper we will give a complete qualitative discussion of the shunted-junction model with a dc current bias $\left(I_{\mathrm{ac}}=0\right.$, the autonomous case). The more important case, $I_{\mathrm{ac}} \neq 0$, (the nonautonomous case) will be discussed in the following paper..$^{11}$ Our aim is twofold; first, to give an understanding of the junction behavior at various points in the parameter space, and at the same time to divide the parameter space into regions of qualitatively different junction behavior. Second, the discussion presented below will introduce some of the mathematical concepts and methods which will be further elaborated in the more complicated nonautonomous case. ${ }^{11}$ Section II contains the mathematical foundation for our present discussion. The same differential equation [Eq. (1)] has been discussed extensively in the literature on so diverse subjects as automatic frequency control, ${ }^{12}$ electric machines, ${ }^{13}$ satellite motion, ${ }^{14}$ and the classical pendulum,${ }^{15}$ and all the mathematical theorems necessary for our purpose may be found in the literature. In Sec. III an interpretation of the mathematical results will be given in terms of the Josephson-junction behavior with particular emphasis on how the parameter values affect the dc current-voltage characteristic. Finally, Sec. IV gives the summary and conclusion of the present paper.

\section{THEORY}

For the purpose of the present paper where the discussion of Eq. (1) will be given mainly with reference to the phase plane $(\phi, \dot{\phi})$ it is convenient to write Eq. (1) in the form

$$
\begin{aligned}
& \dot{\phi}=y, \\
& \dot{y}=\rho+\alpha \sin \Omega \tau-(1 / \sqrt{\beta})(1+\epsilon \cos \phi) y-\sin \phi,
\end{aligned}
$$

where $\rho=I_{\mathrm{dc}} / I_{0}, \alpha=I_{\mathrm{ac}} / I_{0}, \beta=2 e I_{0} C / \hbar G^{2}, \Omega=\omega / \omega_{0}$, and time is measured in units of $1 / \omega_{0}$ where $\omega_{0}$ $=\left(2 e I_{0} / \hbar C\right)^{1 / 2}$. Here the dot means differentiation with respect to normalized time.

As already mentioned above there exist parameter values for which the dc $I V$ curve (i.e., the dependence of $\langle\dot{\phi}\rangle$ on $\rho$ ) is double valued. For this reason, and for reasons to become obvious in the nonautonomous case to be discussed in the companion paper,$^{11}$ it is important to consider the initial-value problem. Thus for a certain value of the parameter $\rho$ we are interested in the solution $y\left(\tau, \phi_{0}, y_{0}, \tau_{0}\right)$, where $\phi_{0}, y_{0}$ are initial conditions at time $\tau_{0}$.

It is known that any solution to Eq. (3) can be written in the form

$$
\begin{aligned}
\dot{\phi}\left(\tau, \phi_{0}, y_{0}, \tau_{0}\right)= & y_{\mathrm{tr}}\left(\tau, \phi_{0}, y_{0}, \tau_{0}\right) \\
& +y^{*}\left(\tau, \phi_{0}, y_{0}, \tau_{0}\right),
\end{aligned}
$$

where $y_{\mathrm{tr}}\left(\tau, \phi_{0}, y_{0}, \tau_{0}\right)$ is the transient response with the property that it goes to zero as $\tau$ goes to infinity, and $y^{*}\left(\tau, \phi_{0}, y_{0}, \tau_{0}\right)$ is a stable steadystate solution, which is the recurrent trajectory of Eq. (3) in its phase space (an equilibrium point, a periodic or almost periodic motion). Hence we find

$$
\left\langle\dot{\phi}\left(\tau, \phi_{0}, y_{0}, \tau_{0}\right)\right\rangle=\left\langle y^{*}\left(\tau, \phi_{0}, y_{0}, \tau_{0}\right)\right\rangle,
$$

where \langle\rangle denotes time average. The dc voltage $V$ being proportional to $\langle\dot{\phi}\rangle$ is hence determined by the stable steady states, and, if more than one stable steady state exists, also by the initial conditions, and of course on the parameters $\alpha, \beta, \epsilon$, $\Omega$, and $\rho$. The number of branches to be expected in the $I V$ curve depend on the number of steadystate solutions.

Thus, in order to obtain the $I V$ curve it is necessary todetermine: (i) all the stable states of the system, (ii) the regions of attraction of these states, and (iii) the range of initial conditions. This leads us to the standard problem of the qualitative theory of dynamical systems, the division of the system [Eq. (3)] phase space by the steadystate trajectories and separatrices, and the decomposition of the parameter space into domains corresponding to the qualitatively different phasespace structures.

In the present paper we consider the autonomous case of Eq. (3), i.e., $\alpha=0$. The division of the parameter space for this case has been discussed in 


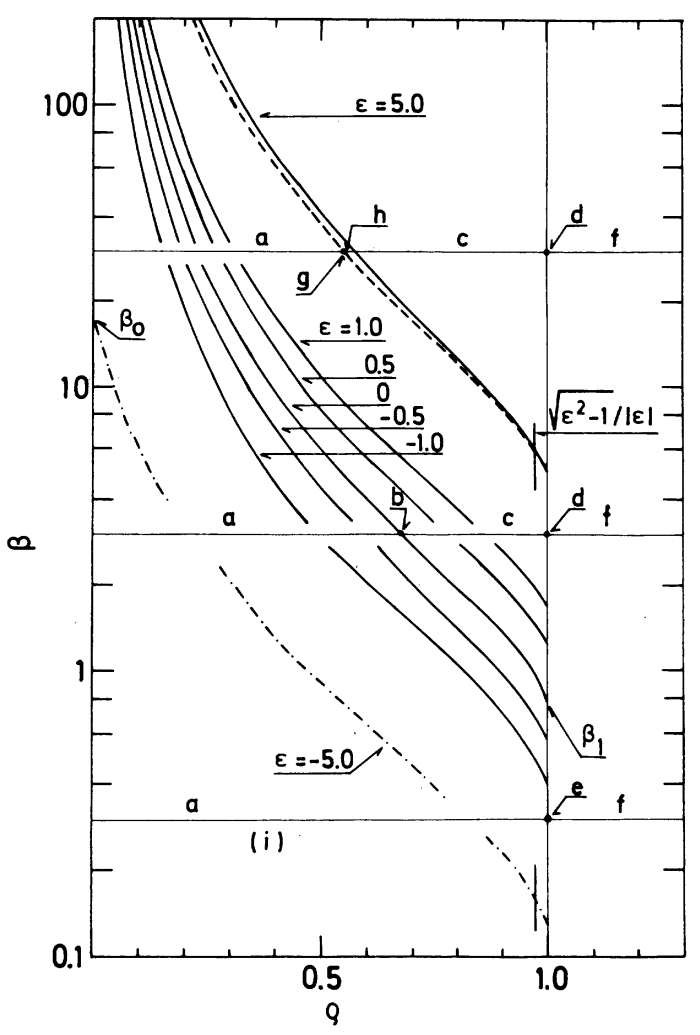

FIG. 1. Parameter space decomposition. The bifurcation curves $\rho=\rho_{c}(\beta, \epsilon)$ are shown by heavy lines for values of $\epsilon$ as indicated. The dashed line shows the curve $\rho=\rho_{d}(\beta, \epsilon)$ for $\epsilon=5$ and the dash-dotted line shows qualitatively the bifurcation for $\epsilon=-5$. The letters correspond to the different pictures in Fig. 2.

the literature on phase-locked loops ${ }^{12,16}$ and on Josephson junctions. ${ }^{2,3}$ The bifurcation curves decomposing the parameter space are shown in Fig. 1 in the $\beta, \rho$ plane for several values of $\epsilon$. The curves, to be denoted $\rho=\rho_{c}(\beta, \epsilon)$ below, are calculated numerically (the curve for $\epsilon=-5$ is, however, only qualitative), and simple approximate expressions are given in Appendix A. In what follows, the structure of the solutions to Eq. (3) will be discussed as $\rho$ is varied while $\beta$ and $\epsilon$ are kept constant. Three values of $\beta$ have been chosen in Fig. 1, indicated by the horizontal lines marked with lower case letters which pass through characteristic domains in the parameter space (the letters on these lines indicate which of the correspondingly marked phase portraits in Fig. 2 apply to the point or region in question).

For $|\epsilon|<1$ there are two and only two qualitatively different cases to consider. One corresponding to large and intermediate values of $\beta$ and the other corresponding to small $\beta$ [i.e., $\beta<\beta_{1}$, where $\left.\rho_{c}\left(\beta_{1}, \epsilon\right)=1\right]$. In the former case the sys- tem is described by the pendulum equation, ${ }^{15}$ (where $\epsilon=0$, but the behavior in that case is typical for any $|\epsilon|<1$ ). Following the center horizontal line in Fig. 1 for small $\rho$, the phase-plane portrait is the stable equilibrium point shown in Fig. 2(a). As $\rho$ increases and approaches the bifurcation curve [e.g., represented by $\left.\rho_{c}(\beta, 0)\right]$, the stable separatrix loop appears [Fig. 2(b)]. An additional small increase in $\rho$ generates the unique stable rotating limit cycle [Fig. 2(c)] which for $\rho_{c}(\beta, \epsilon)<\rho<1$ co-exists with the stable equilibrium point. Hence, the system has in this region two stable steady states, and the phase plane (which may be folded into a cylinder) is divided into two regions of attraction by the separatrices which pass through the saddle point (the unstable equilibrium point). At a further increment of $\rho$ the stable and unstable equilibrium points approach $\phi=\frac{1}{2} \pi$ from either side, and at $\rho=1$ they merge and vanish through a singular point [Fig. 2(d)], and the system possesses for $\rho>1$ only one stable steady-state solution: the unique rotating limit cycle which attracts the whole phase cylinder [Fig. 2(f)]. The other case for $|\epsilon|<1$ is characterized by $\beta$ small, e.g., at the lower horizontal line in Fig. 1. Here, region (a) persists up to $\rho=1$, i.e., the stable equilibrium point attracts the whole phase cylinder [Fig. 2(a)]. At $\rho=1$ the stable and unstable points merge and the separatrix loop of the singular point is generated [Fig. 2(e)]. A small increase of $\rho, \rho \gtrsim 1$ generates the limit cycle and we get the same portrait [Fig. 2 (f)] as before.

When $\epsilon>1$ (a discussion of this in relation to the Josephson junction will be given in Sec. IV) the
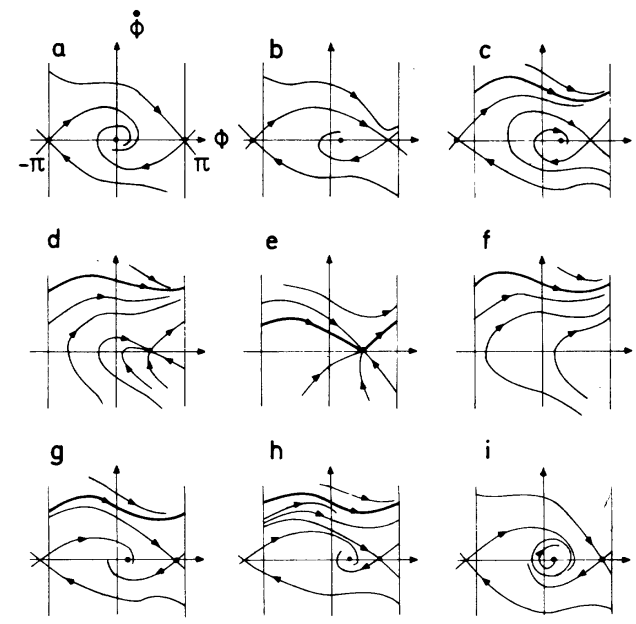

i

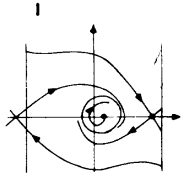

FIG. 2. Qualitative phase-plane portraits. The letters correspond to the regions in Fig. 1 and are discussed in the text. 
behavior of the system differs significantly from the case $|\epsilon|<1$. As shown in $F i g .1$ for $\epsilon=5$, the bifurcation curve splits up into two branches in the region $\rho<\left(\epsilon^{2}-1\right)^{1 / 2} / \epsilon$ (cf Appendix A). The behavior of the system as $\rho$ is varied will in this case be discussed for a-value of $\beta$ corresponding to the upper horizontal line in Fig. 1. As $\rho$ is increased from zero, the system has one stable state, and the equilibrium point attracts the whole phase cylinder [Fig. 2(a)]. As $\rho$ reaches the value corresponding to the dashed curve, to be denoted $\rho_{d}(\beta, \epsilon)$, the degenerate semistable rotating limit cycle appears [Fig. 2(g)]. As $\rho$ is increased further this degenerate limit cycle splits up into two: one stable and one unstable [Fig. 2(h)]. Here the phase cylinder is divided into two regions by the unstable cycle, the upper region being attracted by the stable limit cycle and the lower by the stable point. With a small additional increase in $\rho$ the full curve, $\rho=\rho_{c}(\beta, \epsilon)$, in Fig. 1 is reached and the unstable limit cycle is converted into an unstable separatrix loop (this case is not shown in Fig. 2) which disappears by passing $\rho=\rho_{c}(\beta, \epsilon)$ so that the phase portrait becomes the one shown in Fig. 2(c). As $\rho$ is further increased the behavior of the system is qualitatively the same as for $|\epsilon|<1$ : the equilibrium points merge when $\rho=1$ [Fig. 2(d)] and the unique stable eycle attracting the whole cylinder exists for $\rho>1$. The case $\beta<\beta_{1}$ is the same as if $|\epsilon|<1$.

For the region $\epsilon<-1$ (the physical relevance will be discussed in Sec. IV), the behavior of the system is similar to the case $|\epsilon|<1$ with one essential difference, ${ }^{16}$ in the interval $0 \leq \rho<\left(\epsilon^{2}\right.$ $-1)^{1 / 2} /|\epsilon|$ the equilibrium point $y=0, \phi=\sin ^{-1} \rho$ is unstable and is embraced by the oscillating stable limit cycle so that the system has the phase portrait shown in Fig. 2(i) rather than in Fig. 2(a) for small $\rho$. This oscillating cycle is compressed as $\rho$ increases but does not disappear until $\rho=\left(\epsilon^{2}\right.$ $-1)^{1 / 2} /|\epsilon|$. When $\rho>\left(\epsilon^{2}-1\right)^{1 / 2} /|\epsilon|$ the system behaves as in the $|\epsilon|<1$ case. If, furthermore, $\epsilon$ $<-3$ the bifurcation curves have the additional feature that $\rho_{c}\left(\beta_{0}, \epsilon\right)=0$ is satisfied by a finite value of $\beta=\beta_{0}$ as seen in Fig. 1 for $\epsilon=-5 .^{16}$ The discussion above still applies for $\beta<\beta_{0}$, i.e., the stable equilibrium point in Figs. 2(a), 2(b), and 2(c) has become unstable and instead the stable oscillatinglimit cycle has appeared. For $\beta>\beta_{0}$, however, the system has only the rotating stable solutions. ${ }^{16}$

\section{IV CURVES}

In the interval $0 \leqslant \rho<1$, the bifurcation curves separate the parameter space (cf. Fig. 1) into two regions. Denoting the bifurcation curves by $\rho=\rho_{c}(\beta, \epsilon)$ we have that for $\rho \geq \rho_{c}(\beta, \epsilon)$, the trajectories on the phase cylinder are attracted by one of two steady-state solutions depending on the imposed initial conditions. One of these solutions corresponds to finite voltages, and the other to zero voltage. The zero-voltage branch is explained as follows. For values of $\epsilon \in(-1, \infty)$ and for any $\beta$, one steady state is the stable equilibrium point implying that the $I V$ curve always has a branch $V=0$ for $0 \leq \rho<1$. This is the case also for $\epsilon \in(-3,-1)$, even though the equilibrium point in the region $0 \leq \rho \leq\left(\epsilon^{2}-1\right)^{1 / 2} /|\epsilon|$ is unstable because the oscillating stable-limit cycle [Fig. $2(i)]$ satisfies $V=\left(\hbar \omega_{0} / 2 e\right)\langle\dot{\phi}\rangle=0$. Only for the case $\epsilon<-3$ and $\beta>\beta_{0}$ (Fig. 1) the zero-voltage solution does not exist.

The finite-voltage branch of the $I V$ curve has the following properties: For $|\epsilon|<1$ or $\epsilon>1$ and $\rho$ $>\left(\epsilon^{2}-1\right)^{1 / 2} / \epsilon$ the voltage is a continuously increasing function of $\rho$ for all values of $\rho>\rho_{c}(\beta, \epsilon)$. For $|\epsilon|<1$, the voltage curve terminates at $V=0$, with zero slope at $\rho=\rho_{c}(\beta, \epsilon)$. For $\epsilon>1$ and $\rho<\left(\epsilon^{2}\right.$ $-1)^{1 / 2} / \epsilon$ the voltage curve terminates at a finite voltage, $V>0$, also with zero slope. This is understood by the following arguments. The voltage $V$ is determined by the equation

$$
V=\left(\hbar \omega_{0} / 2 e\right)\langle\dot{\phi}\rangle=\left(\hbar \omega_{0} / 2 e\right)[2 \pi / T(\rho)],
$$

where $T(\rho)$ is the dimensionless time period of the stable-rotation limit cycle.

For $|\epsilon|<1$ or $\epsilon>1$ and $\rho>\left(\epsilon^{2}-1\right)^{1 / 2} / \epsilon$ the condition $\rho=\rho_{c}(\beta, \epsilon)$ corresponds to the separatrix loop [Fig. 2(b)] in the phase space. Since the time period of the separatrix loop is infinite, i.e.,

$$
\lim _{\rho \rightarrow \rho_{c}} T(\rho)=\infty,
$$

the finite-voltage branch of the $I V$ curve has its end point at $V=0$ when $\rho=\rho_{c}(\beta, \epsilon)$. Furthermore,

$$
T_{\rho}^{\prime}(\rho)<0 \text { for } \rho>\rho_{c}(\beta, \epsilon),
$$

since the limit cycle moves to larger values of $y$ when $\rho$ is increased..$^{16}$ Since from Eqs. (5) and (7)

$$
V_{\rho}^{\prime}(\rho)=-\left(\hbar \omega_{0} / 2 e\right)\left[2 \pi T_{\rho}^{\prime}(\rho) / T^{2}\right]>0,
$$

the finite-voltage branch always has a positive slope. In the limit $\rho \rightarrow \rho_{c}(\beta, \epsilon)$ the stable-limit cycle approaches the saddle point and the period $T(\rho)$ is mainly determined by the limit-cycle motion in the neighborhood of this point. Using this we find that for $0<\rho-\rho_{c}(\beta, \epsilon) \ll 1$ the period $T(\rho)$ is defined by the equation

$$
\rho-\rho_{c}(\beta, \epsilon)=k e^{-\alpha T(\rho)},
$$

where $k$ and $\alpha$ are positive constants. From Eq. (9) we find

$$
T_{\rho}^{\prime}(\rho)=-(1 / k \alpha) e^{\alpha T} .
$$




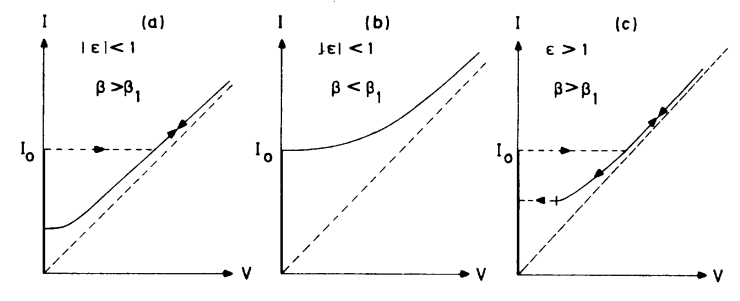

FIG. 3. Typical $I V$ curves.

Inserting Eq. (10) in Eq. (8) we find that $V_{\rho}^{\prime}(\rho) \rightarrow \infty$ as $T \rightarrow \infty$, which implies that the finite-voltage branch has zero slope at $V=0$. Two typical examples of $I V$ curves corresponding to $\beta>\beta_{1}$ and $\beta<\beta_{1}$ are shown in Figs. 3(a) and 3(b), respectively.

For $\epsilon>1$ and $\rho<\left(\epsilon^{2}-1\right)^{1 / 2} / \epsilon$ the endpoint of the finite-voltage branch is due to the degeneration of the stable limit cycle [Fig. 2(g)] at a finite mean value of $\dot{\phi}$. This happens for decreasing $\rho$ when $\rho=\rho_{d}(\beta, \epsilon)$ shown in Fig. 1 is reached; also in this case the slope of the $I V$ curve at the end point is zero. The corresponding $I V$ curve is shown in Fig. 3(c). Finally, for $\epsilon<-1$ and $\beta<\beta_{0}$ the behavior of the finite-voltage branch is similar to that for $|\epsilon|<1$. Approximate expressions for the $I V$ curves are given in Appendix $B$.

Voltage jumps in the $I V$ curve from one branch to another are either due to the degeneration of the steady-state solution or due to the random jumps in the junction coordinates $(\phi, y)$ when, for example, a steady-state solution is not far from the boundary of its region of attraction in the phase space. The first type of jump occurs either when the system is locked by the equilibrium point and $\rho$ is increasing towards 1 [Fig. 3(a), (b), and (c)], or, if $\epsilon>1$, when the system is locked by the limit cycle and $\rho$ is decreasing towards $\rho=\rho_{d}(\beta, \epsilon)$ [Fig. 3(c)]. The second type of jump can take place when the system with $\rho<1$ is locked by the rotating limit cycle; as $\rho$ is decreased towards $\rho_{c}(\beta, \epsilon)$ a jump from the limit cycle to the region of attraction of the stable point (which is close to the cycle) may occur, caused by a small perturbation of the system. This latter possibility is not shown in Fig. 3. The $I V$ curve has no voltage jumps from one branch to another when the degeneration of the steady state coincides with the generation of another steady state with the same mean value of $\dot{\phi}$. This is the case for $\beta<\beta_{1}$ as shown in Fig. 3(b).

\section{SUMMARY AND CONCLUSION}

In the preceding paragraph the behavior of the shunted junction model has been discussed for ar- bitrary values of the parameters. This has been done without imposing limitations on their magnitudes. While $\beta$ may have any value, we know from the Hamiltonian calculations on tunnel junctions $^{8}$ that $0<\epsilon<1$. On the other hand for other types of junctions the physical mechanism giving rise to the so-called $\cos \phi$ term may be different and at present very little can be said about the magnitude of $\epsilon$. All experiments apparently gives a value of $\epsilon$ close to $-1 .^{10}$ From the previous discussion it can safely be argued that $\epsilon<-1$ is unphysical since the system will go into oscillation for $0 \leq \rho<\left(\epsilon^{2}-1\right)^{1 / 2} /|\epsilon|$, and hence power would be dissipated without supplying energy to the system. The case $\epsilon>1$ is not a priori unphysical. If this occurs, the experiment to check it is in principle simple; it can be verified directly from the IV curve which should look qualitatively similar to Fig. 3(c). However, this kind of experiment may not be so simple to interpret since it may be difficult to distinguish between the jump in Fig. 3(c), and a jump generated by a noise perturbation as discussed in Sec. III. Also other mechanisms may explain the jump which is actually very often observed experimentally. In Fig. 4 is shown two $I V$ curves for the same Sn-O-Sn tunnel junction obtained at different temperatures. The jump to $V=0$ in Fig. 4(a) might be interpreted as evidence for $\epsilon>1$, but actually the jump occurs because the junction was locked to an internal cavity resonance and the lock-range exceeded as evident in Fig. 4 (b) where the cavity induced step is clearly seen. Finally, the hysteresis in the $I V$ curve may have a completely different physical explanation, i.e., be due to self-heating effects ${ }^{17}$ such that the shunted-junction model does not apply at all, or be due to the junction element having a nonsinusoi dal current-phase relation. 4

\section{APPENDIX A: APPROXIMATION FORMULAS FOR THE BIFURCATION CURVES}

When the parameters $1 / \sqrt{\beta}$ and $\rho$ are small the system Eq. (3), is close to the conservative system

$$
\dot{\phi}=y, \quad \dot{y}=-\sin \phi
$$

with the integral $H(\phi, y)=\frac{1}{2} y^{2}-\cos \phi=h$. Hence, perturbation theory can be used to obtain an approximation of the bifurcation curves. Using Pontrjagin's formula ${ }^{18}$ for the averaging, the system can be transformed to the form

$\frac{d h}{d t}=\int_{-\pi}^{\pi}\left[\rho-\frac{1}{\sqrt{\beta}}(1+\epsilon \cos \phi)(2 h+\cos \phi)^{1 / 2}\right] d \phi \equiv \psi(h)$

for $h \geq 1$.

The root of the equation $\psi(h)=0, h^{*}$, satisfying 


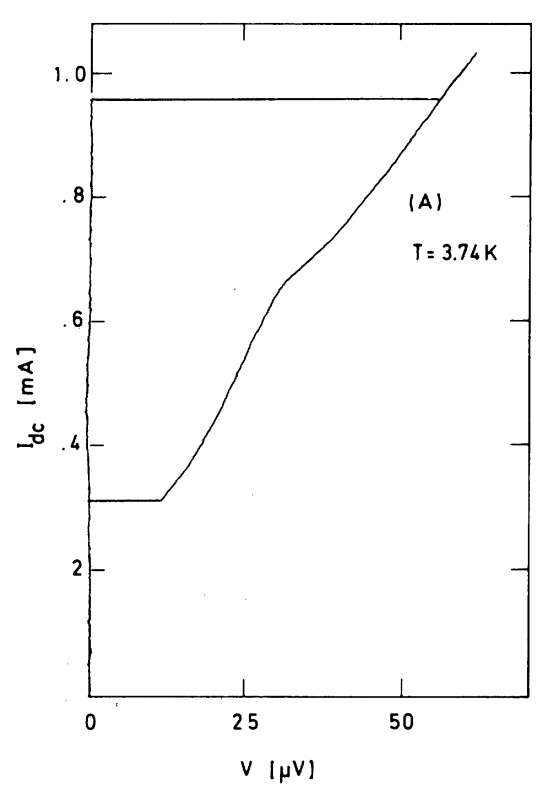

the condition $d \psi /\left.d h\right|_{h=h} *<0$ determine the curve

$$
H(\phi, y)=h^{*}
$$

which is close to the unique stable-limit cycle for $y>0$. The condition $h^{*}=1$ gives the approximate formula for the bifurcation curve

$$
\rho_{c}=\frac{4}{\pi \sqrt{\beta}}\left(1+\frac{\epsilon}{3}\right)\left\{\begin{array}{l}
-3<\epsilon<1 \\
\epsilon>1, \quad \rho>\frac{\left(\epsilon^{2}-1\right)^{1 / 2}}{\epsilon}
\end{array}\right.
$$

which was also derived in Ref. 3. This formula gives a very good approximation for the bifurcation curve in the case $-3<\epsilon<1$ almost for the whole range of $\beta$. For $\epsilon<-3$, it is invalid. In the case, $\epsilon>1$, where the bifurcation curve splits up in the region $\rho<\left(\epsilon^{2}-1\right)^{1 / 2} / \epsilon$ Eq. (A4) is still reasonable for the full curve in Fig. 1, another approximation, well known in the theory of automatic phase control systems, ${ }^{19}$ should, however, be used for the dashed curve

$$
\rho_{d}=[\epsilon(2 \beta-\epsilon)]^{1 / 2} / \beta, \epsilon>1, \rho<\left(\epsilon^{2}-1\right)^{1 / 2} / \epsilon .
$$

\section{APPENDIX B: APPROXIMATIONS FOR THE IV CURVES}

In the limit of small $\beta$, Eq. (3) may be transformed to

$$
(1+\epsilon \cos \phi) \dot{\phi}+\sin \phi=\rho,
$$

where the time is now measured in units of $1 / \omega_{c}$ and $\omega_{c}=2 e I_{0} / \hbar G$. tion

$$
\begin{aligned}
& t=f(\phi)+C, \\
& \begin{aligned}
f(\phi)=\frac{2}{\left(\rho^{2}-1\right)^{1 / 2}} \arctan \frac{\rho \tan \frac{1}{2} \phi-1}{\left(\rho^{2}-1\right)^{1 / 2}} \\
\quad \times \in \ln |\rho-\sin \phi|,
\end{aligned}
\end{aligned}
$$

where $C$ is defined by the initial conditions.

According to Eq. (B1), for all $|\epsilon|<1, f(\phi)$ is an increasing function of $\phi$. Hence the period of rotation, i.e., the time $T$ corresponding to a phase increment of $2 \pi$, can be determined from the equations

$$
0=f(\pi+0)+C, \quad T=f(3 \pi-0)+C .
$$

Solving Eqs. (B2) and (B3) we obtain for the period of rotation $T$

$$
T=2 \pi /\left(\rho^{2}-1\right)^{1 / 2}
$$

and the formula for the $I V$ curve is

$$
V=\frac{\hbar \omega_{c}}{2 e} \frac{2 \pi}{T}=\frac{\hbar \omega_{c}}{2 e}\left(\rho^{2}-1\right)^{1 / 2}\left\{\begin{array}{l}
|\epsilon|<1 \\
\beta \ll 1
\end{array}\right.
$$

which is identical to the well known formula for the $\epsilon=0$ case.

For small $\beta$ and $\epsilon>1$ the formula

$$
\begin{aligned}
V= & \frac{\hbar \omega_{c}}{2 e}\left(\rho^{2}-1\right)^{1 / 2} \\
& \times\left(1-\frac{1}{\pi} \arctan \frac{1}{\rho}\left[\left(\epsilon^{2}-1\right)\left(\rho^{2}-1\right)\right]^{1 / 2}\right)^{-1}
\end{aligned}
$$


can be derived.

For large $\beta$, when the system is close to being a conservative system the approximation for the $I V$ curve can be determined by Eq. (5) with

$$
T(\rho)=\int_{-\pi}^{\pi} \frac{d \phi}{2\left(h^{*}+\cos \phi\right)}
$$

where $h^{*}>1$ is defined by Eqs. (A2) and (A3).
*Permanent address: Scientific-Research Institute of Applied Mathematics and Cybernetics, Gor'kii University, Gor'kii, USSR.

${ }^{1}$ W. C. Stewart, Appl. Phys. Lett. 12, 277 (1968).

${ }^{2}$ D. E. McCumber, J. Appl. Phys. $\overline{39}, 3113$ (1968).

${ }^{3}$ W. A. Schlup, J. Phys. C 7, 736 (1974).

${ }^{4}$ A. Baratoff, J. A. Blackbưrm, and B. B. Schwartz, Phys. Rev. Lett. 25, 1096 (1970); Erratum, 1738 (1970); F. Auracher and T. van Duzer, Appl. Phys. Lett. 21, 515 (1972).

${ }^{5}$ N. F. Pedersen and K. Saermark, Physica (Utr.) 69, 572 (1973).

${ }^{6}$ W. C. Stewart, J. Appl. Phys. 45, 452 (1974).

${ }^{7}$ B. D. Josephson, Phys. Lett. 1,251 (1962); Adv. Phys. 14, 419 (1965).

${ }^{8}$ N. R. Werthamer, Phys. Rev. 147, 255 (1966); U. K. Poulsen, Rev. Phys. Appl. 9, 41 (1974); R. E. Harris, Phys. Rev. B 10, 84 (1974).

${ }^{9}$ L. G. Aslamasov and I. A. Larkin, JETP Lett. 9, 87 (1969); H. Hojgård Jensen and P. E. Lindelof, J. Low Temp. Phys. 23, 469 (1976); B. S. Deaver, B. G. Boone and R. Rifkin, Phys. Lett. A 57, 186 (1976). ${ }^{10}$ N. F. Pedersen, T. F. Finnegan, and D. N. Langenberg, Phys. Rev. B 6 , 4151 (1972). C. M. Falco,
W. H. Parker, and S. E. Trullinger, Phys. Rev. Lett. 31,933 (1973). D. A. Vincent and B. S. Deaver, ibid. $\overline{32}, 212(1974)$.

${ }^{11}$ V. N. Belykh, N. F. Pedersen, and O. H. Soerensen, Phys. Rev. B 16, 4860 (1977), following paper.

${ }^{12}$ Phase Synchronization, edited by L. N. Belynstina and V. V. Shakhgil'djan (Svyaz', Moscow, 1975) (in Russian), Chaps. 2, 3, 5, and 6 .

${ }^{13} \mathrm{~J}$. Stoker, Nonlinear Vibrations in Mechanical and Electrical Systems (Interscience, New York, 1950).

${ }^{14} \mathrm{Yu}$. A. Sadov, Cosm. Res. 7, 45 (1969).

${ }^{15}$ F. Tricomi, Ann.R. Scuola, Normale Superiore di Pisa, Ser. 11, 2, 1 (1933); A. Andronov, A. Vitt, and S. E. Khaikin, Theory of Oscillations (Pergamon, New York, 1966).

${ }^{16}$ L. N. Belynstina and V.N. Belykh, Diff. Equat. 9, 309 (1973).

${ }^{17}$ W. J. Skocpol, M. R. Beasley, and M. Tinkham, J. Appl. Phys. 45, 4054 (1974).

${ }^{18}$ L. S. Pontrjagin, Zh. Fiz. (USSR) 6, No. 1-2 (1934); N. N. Bautin, Appl. Math. and Mech. PMM. 34, 812 (1970).

${ }^{19}$ M. Brunk, Arch. Elekt. Übertr. 19, 649 (1965). 